\title{
Quelques Installations d'Accumulation par pompage récemment réälisées en France et à l'Etranger
}

\author{
Par M. MARTIN, Ingénieur en chef à la Compagnie de Construction mécanique "Procédés Sulzer"
}

(SUITE ET FIN)

USINE DE BELLEVILLE DE LA SOCIETEE D'ELECTROCHIMIE, D'ELECTRO-METALLURGIE ET IDES ACIE-

\section{RIES ELECTRIQUES D'UGINE.}

L'installation réalisée dans le Dauphiné par la Société d'Electro-Chimie, d'Electro-Métallurgie et des Acieries Electriques d'Ugine dépasse de loin celle de Munster par la puissance des unités mises en ceuvre et celle de Viverone par la hauteur séparant le réservoir supérieur de l'Usine de pompage. Elle est remarquable, en outre, par le rendement auquel elle aboutit.

L'important domaine hydroélectrique dont est concessionnaire cette Société dans les bassins de l'Arly, du Bonnant et du Doron de Beaufort comporte sur ce dernier cours d'eau toute une série d'usines étagées entre Venthon, au confluent de l'Arly et du Doron, et Belleville, à l'origine de la vallée du Dorinet, affluent de droite du Doron de Beaufort (photo 16).

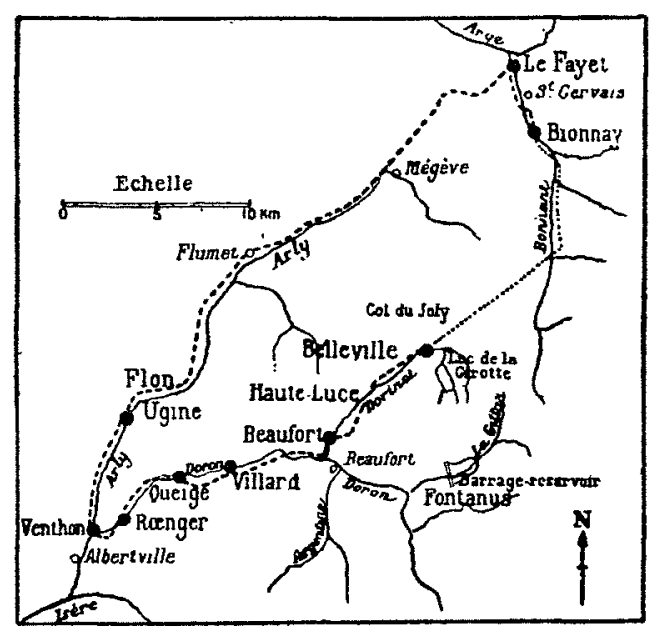

Fig. 16. - Plan général des Usines du domaine hydraulique de la Société d'Electrochimie, d'Electrométallurgie et des Aciéries électriques d'Ugine (Saroie).

Pour la régulation journalière, on a pu utiliser les bassins d'accumulation que constituent les tunnels d'amenée des différentes usines. Pour les usines du Doron de Beaufort seulement, ces tunnels représentent environ 32 kilomètres de longueur et permettent une accumulation de 25 millions de mètres cubes utilisables sur une hauteur moyenne de 650 mètres, soit environ 30.000 .000 kilowatts-heure.

Pour la régulation annuelle, il était intéressant de pouvoir trouver des réservoirs d'accumulation situés aussi haut que possible et représentant, par conséquent, la plus grande puissance possible par $\mathrm{m}^{3}$ d'eau accumulée.

Le lac de la Girotte, qui se trouve sur le versant gauche du Dorinet, affluent du Doron de Beaufort, se prêtait avantageusement à ce service. Situé à 1.724 mètres d'altitude, il a une. capacité de 30 millions de mètres cubes et une profondeur de
100 mètres. Sa cuvette est étanche et, ne recevant aucun cours d'eau important, il ne présente pas de risque d'envasement.

Percé à 80 mìtres de profondeur, il permet l'utilisation d'une réserve de 28 millions de mètres cubes à cles altitudes variant de 1.724 à 1.644 mètres.

Ce volume n'est pas seulement utilisé par l'Usine de Belleville, la dernière à l'amont dans le bassin du Doren de Beaufort, mais par toutes les usines qui s'échelonnent au long de la rivière, jusqu'à Venthon, au voisinage d'Albertville, soit sous une chulte totale d'environ 1.300 mètres. Ce réservoir représente ainsi à lui seul une énergie de 60 millions de kilowatts-heure.

Toutefois, comme le bassin versant du lac de la Girotte ne représente qu'un apport annuel de 6 millions de mètres cubrs d'eau environ, il est nécessaire, pour utiliser la capacité totah du lac, de chercher ailleurs 22 millions de mètres cubes.

14 millions sont obtenus par dérivation dans le lac de divers ruisseaux environnants.

L'usine de pompage de Belleville fournit les $\cdot 8$ millions de mètres cubes supplémentaires.

La disposition générale de l'installation est schématisée par le profil en long de la vue (photo 17) qui va passer sous v0: yeux.

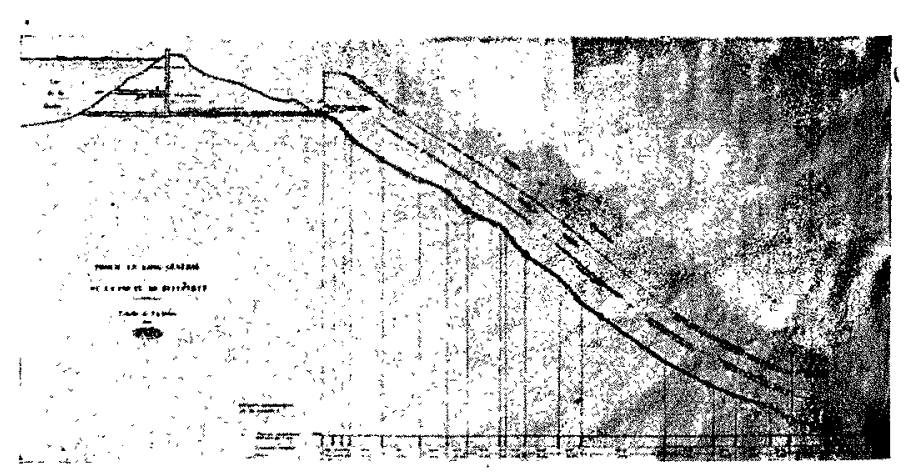

Fig. 17. - Société d'Electrochimie et d'Electrométallurgie, profil en long général de l'installation de Belleville.

V'ous pouvez vous y rendre compte tout d'abord de la facon dont a été exécuté le percement du lac, travail qui s'est exécut' en deux stades. On a d'abord percé la galcrie d'amenée et le puits vertical donnant accès aux deux galeries transversales qui ont permis de venir percer le lac à deux profondeurs diffét rentes, d'une part à la cote 1675 , d'autre part à la cote 1644 .

Cette dernière opération a été terminée cette année et fait le plus grand honneur aux ingénieurs de la Société d'Electro Chimie, d'Electro-Métallurgie ct des Aciéries Electriques d'Ugina qui l'ont menée à bonne fin.

On voit donc que c'est une lame d'eau de $80 \mathrm{~m}$. (entre les cotes 1724 et 1644) que l'on pourra utiliser et qui correspónd aux 22 millions de mètres cubes cités tout à l'henre. 
Le lac est relié à l'Usine de Belleville par une conduite forcée comportant les sections suivantes :

360 mètres en une conduite de $1 \mathrm{~m}$. de diamètre.

Suivis de :

160 mètres en 2 conduites parallèles de 0,75

$125 \quad \cdots \quad-\quad-\quad \quad-\quad$ de 0,64

210 - $\quad$ - $\quad$ de 0,600 environ et enlin 45 - $\quad$ - $\quad$ r $\quad-\quad$ de 0,800

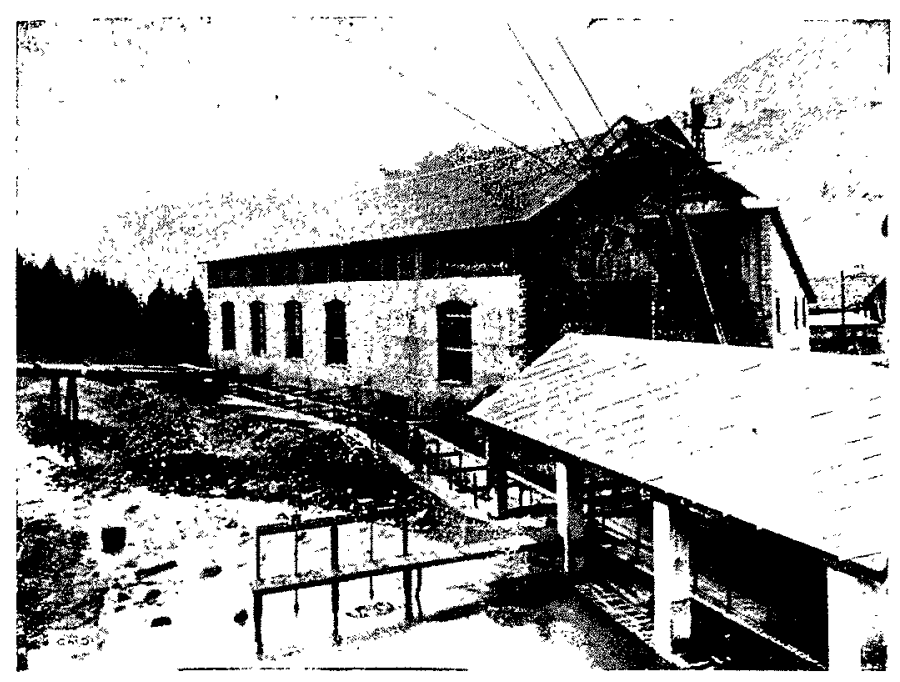

Fig. 18. - Société d'Electrochimie et d'Electrométallurgie. Usine de Belleville, vue extérieure.

Ces conduites desservent directement les deux groupes à double effet de 5.000 chevaux que comporte l'Usine de Belleville installée à la cote 1213 .

La vue extérieure de l'Usine (photo 18) vous permet de vous rendre compte de sa disposition générale. Vous apercevez tout d'abord l'arrivée des deux conduites forcées desservant chacune

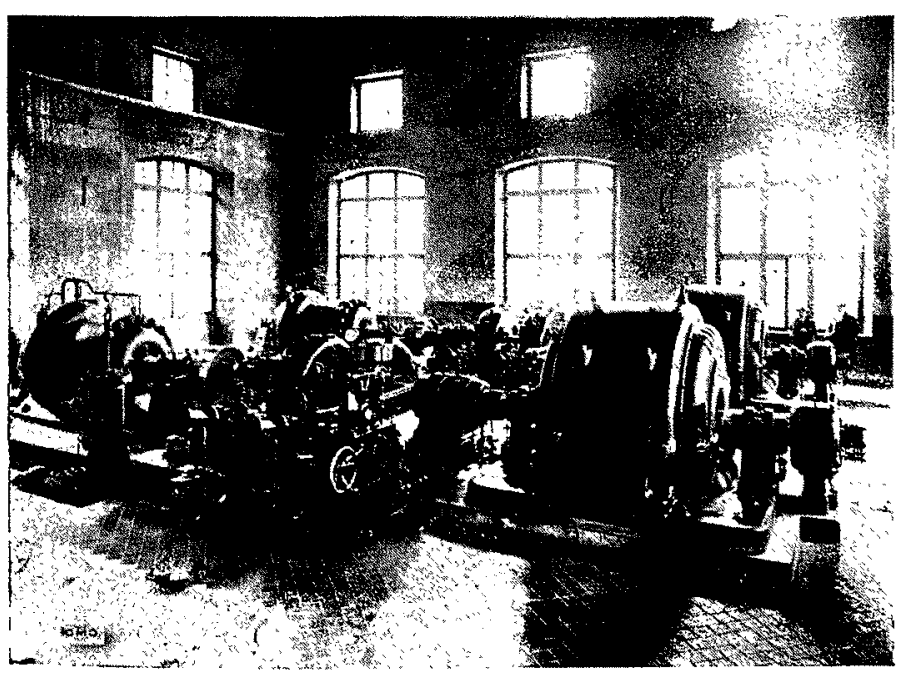

Fig. 19.- Société d'Elcctrochinie et d'Electrométallurgie Usine de Belleville, vue générale intérieure.

l'un des groupes à double effet. Au premier plan, le cours du Dorinct qui alimente, à l'aide d'un barrage situé un peu à l'amont, tout un systeme de désableurs DUFoUR qui longe toute la laçade postérieure pour venir alimenter le puisard d'aspiration des pompes centrifuges lequel tient lieu également de bassin de fuite des turbines. Ces désableurs permettent un nettoyage rigoureux de l'eau avant son accès aux pompes - précaution indispensable si l'on songe que celles-ci ne sont mises en route que lorsque les eaux sont hautes et chargées de sable et détritus de toute sorte.

En avant du pignon, vous apercevez le poste inférieur du câble transbordeur mettant en relation l'usine et les chantiers d'aménagement du lac et de montage des conduites forcées et qui fut, en quelque sorte, la cheville ouvrière de toute l'installation.

Sur la photo $n^{\mathrm{e}} 19$ qui représente l'ensemble de l'intérieur de

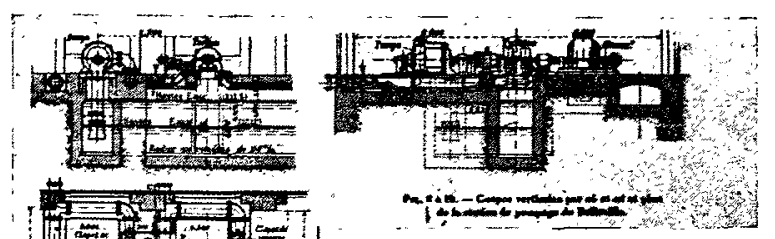

Fig. 20.- Société d'Elec trochimie et d'Electrométallurgie. Usine de Belleville, plan des Groupes moto-turbo pompes de $5.000 \mathrm{Cr}$.

l'Lsine, vous voyez se projeter les deux unités à double effet de 5.000 chevaux.

Chacune comporte, au centre, une roue Pelton comportant en parallèle deux roues à un ajutage. Elle est accouplée, d'une part à un alternateur de $3.800 \mathrm{Kva}, 1.000$ tours, d'autre part, à une pompe centrifuge Sulzer capable d'un débit horaire moyen de $1.600 \mathrm{~m}^{3}$ à $500 \mathrm{~m}$. de hauteur moyenne. L'alternateur comportant une excitatrice en bout d'arbre n'a pu, comme à Vive-

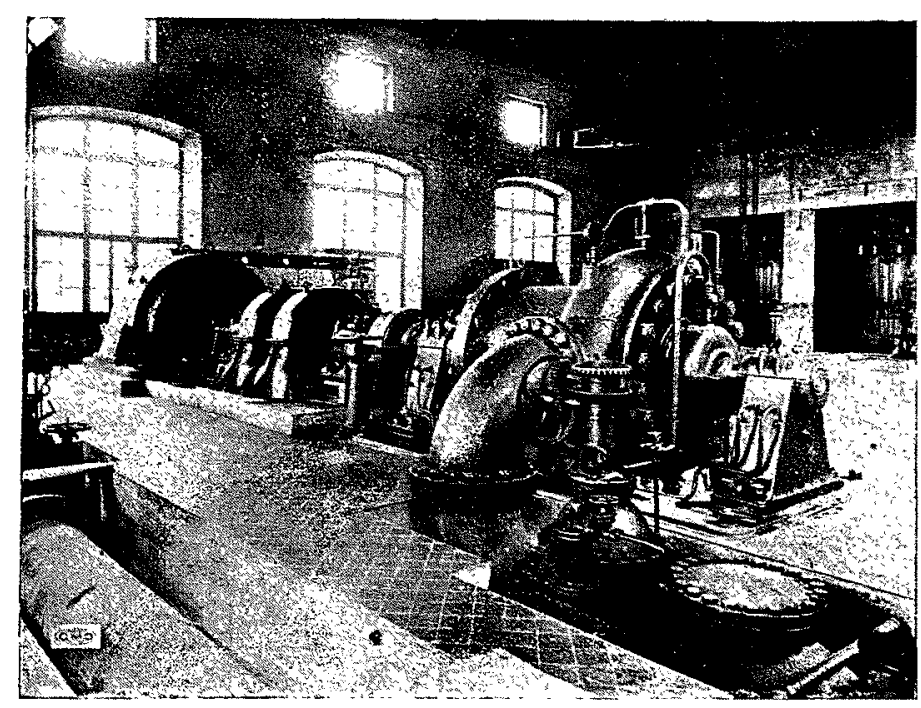

Fig. 21. - Société d'Electrochimie et d'Electrométallurgie. Usine de Belleville, vue de la Pompe de $5.000 \mathrm{CV}$ avec ses appareils de protection et de réglage.

rone, ètre placé entre la pompe et la turbine, en sorte qu il n'y a cu'un manchon d'accouplement débrayable entre pompe et turbine et que lors du fonclionnement du groupe en pompe, colle-ci est entrainée par l'alternateur à travers la turbine qui tourne alors à vide. 
Sur la rue $n^{\circ} 20$ representant l'installation en plan ef en coupe, vous distinguez la disposition relative des trois unités et les conditions dans lesquelles se fait l'aspiration des pompest sans interposition de clapet de pied. L'amorgage se fait par éjecteur. On a pu ainsi réluire au minimum les pertes de charge à l'aspiration.

Iu refoulement (photo 21), chaque pompe est protégée contre les coups de bélier par un clapet a by-pass amortisseur du genre do celui decrit: lout à l'heure à propos de l'installation de Viverone. Aree une seclion de passage du by-pass demviron 1/10 de la tuyaterie principale (nous avons ici $125 \mathrm{~mm}$. de diametre au by-pass pour 100 de section au clapet, nous avious à Viverone. 300 contre 800 ) on a constaté, lors du déclenchement brusque de l'alternateur en pleine charge, une surpression denviron $70 \mathrm{~m}$. - soit environ $15 \%$ comme à Viverone - suivant une dépression sensiblement de mème importance et allant ensuite en s'alténuant pas oscillations amorties comme sur le diagramme que j'ai fait passer tout à l'heure sous vos yeux.

Le diagramme des pompes (Photo 22) a été étudié de laçon à pouvoir à la vitesse constante de 1.000 tours/minute, couvrit sans risque de surcharger la pompe ni le moteur, toutes les

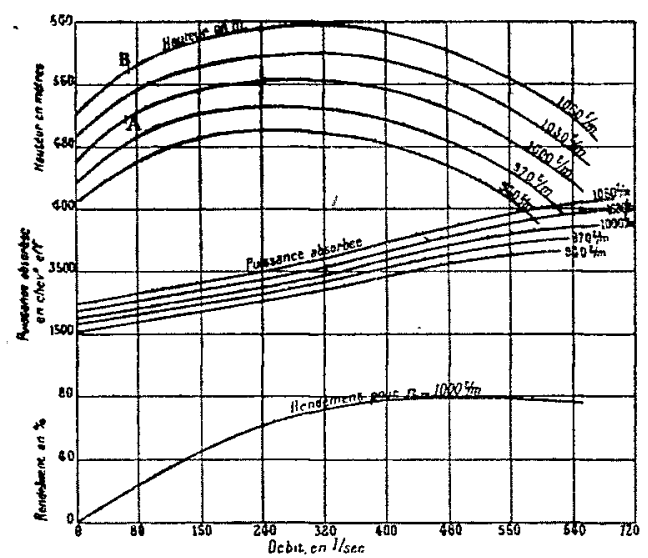

Fig. 22.- Société d'Electrochimie et d'Electrométallurgie. Usine de Belleville. Diagrammes de fonctionnement d'une pompe de $5.000 \mathrm{CV}$.

hauteurs manométriques comprises entre 150 el 530 mètres correspondant aux niveaux extrêmes du lac.

Vous voyez qu'entre ces deux limites les pompes débilent de 600 à 650 litres/seconde sans jamais dépasser la puissance maximum de 5.000 fchevaux.

Au régime moyen de $1.600 \mathrm{~m}^{3} /$ heure, les pompes pourront donc, en 2.500 heures de marche, fournir ensemble les 8 millions de mètres cubes que l'on attend d'elles. La consommation correspondante d'énergie será de 16 millions de $\mathrm{Kw} /$ heure. En tablant sur environ $1 \mathrm{Kw} /$ heure par $\mathrm{m}^{3}$ accumulé à $500 \mathrm{~m}$. de hauteur, c'est 8 millions de $\mathrm{Kw} / \mathrm{h}$. que l'on pourra récupérer, soit un rendement d'environ $50 \%$ ce qui est déjà intéressant, si l'on songe que les 16 millions de $\mathrm{Kw}$ /heure employés à l'accumulation ne coûtent rien, ou à peu près. Mais en fait le résultat est encore plus séduisant. Les 8 millions de $\mathrm{m}^{3}$ accumulés ne sont pas utilisés seulement sous $500 \mathrm{~m}$. de hauteur, mais par toutes les usines échelonnées le long du Doron en'se Belleville et Venthon, sous une chute totale nette d'environ 1.100 mètres. Chaque $\mathrm{m}^{3}$ d'eau accumulée fournit donc, non pas $1 \mathrm{Kw} /$ heure, mais deux, soit au total, environ 17 millions de Kw/heure. En fin de compte, on peut dire, en dépit du paradoxe apparent, que le rendement: d'une telle installation est de $100 \%$ environ.
USINE HYOROELECTHIQUH DE WAGCITH.

Quelpues mots entin, de l'installation de pompenge qule Usines Sulzer de Winterthur viemnent de réaliser pour la Si des Forces Motrices de W'äggital.

Le Wäggilal est mo vallé qui débouche à Siebnen, il trémité orientale du lac de \%urich el a son origine jol) 1m. an sus, environ, au vallon d'Innertal. I)es conditions lopograph particulieresment lavorables ont permis, grâce à la constru d'un barrage de $90 \mathrm{~m}$. de hauteur environ, de créer un late di. mulation de 140 millions de mètres cubes servant. de régulat aux réseaux de Zurich de la Société des Forces molrices du; de la Suisse et des principales usines électriques de Suisse.

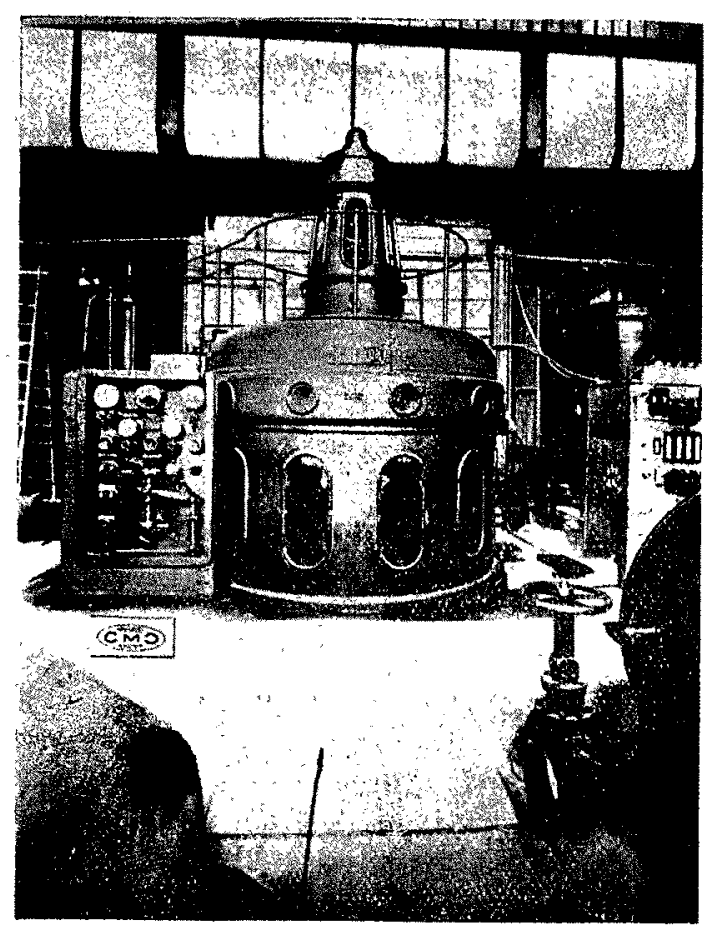

Fig. 23.- Usine de Waggital (Suisse). Groupe moto-pon de $5.500 \mathrm{CV}$ à axe vertical.

La chute totale de $500 \mathrm{~m}$. entre l'Innertal et Siebnen est sée en 2 étages de $250 \mathrm{~m}$. avec une usine inférieure à Sie et une usine intermédiaire $250 \mathrm{~m}$. au-dessus à Rempen. Ch de ces usines comporte, 70.000 chevaux installés, répart 4 unités, soit 140.000 chevaux installés pour l'ensemble deux usines.

L'usine supérieure (de Rémpen) comporte en outre dy moto-pompes destinés, aux périodes d'abondance d'es. utiliser les eaux de surface qui se présentent au nivel Rempen; ces eaux sont recues et peuvent être accumulé moyen d'un barrage de moindre envergure que le barrages rieur et permettant de recueillir $300.000 \mathrm{~m}^{3}$ d'ean enl

Les pompes refoulent les eaux dans le lac artificiel d'In. participant ainsi à son remplissage, parallèlement à son all tation normale.

On a prévu à cet effet 4 groupes moto-pompes (dont? actuellement en route) capables d'assurer ensemble uा de $3 \mathrm{~m}^{3}$ par seconde sous une hauteur de refoulement de 250

Ces groupes sont à axe vertical. Cette disposition a éléin par l'importance de la lame d'eau à épuiser qui alteint environ. Comme il ne fallait pas songer à laire aspirer despo à axe horizontal à une telle hauteur, on s'est résolu à ins 


\section{LA HOUILLE BLANCHE}

des groupes verticaux, les pompes se trouvant à la partie inféricure de l'Usine (photos 23 et 24) et pouvant, éventuellement, fonctionner noyées, en période dé très hautes eaux.

Cette disposition n'a pas permis la réalisalion de groupes à double effet comme ceux de Belleville, de Munster ou de Viverone. L'usine de Rempen comporte donc, nettement séparés, les groupes de pompes et les groujes de turbines.

Les pompes de 5.500 chevaux, les plus importantes, par leurs dimensions, construites jusqu'à ce jour dans le monde enticr comportent 3 étages de roues de $1 \mathrm{~m}$. de diamètre assurant un débit de 750 litres/seconde. Elles tournent à 750 tours accouplées directement à des moteurs asynchrones synchronisés Oerlikon.

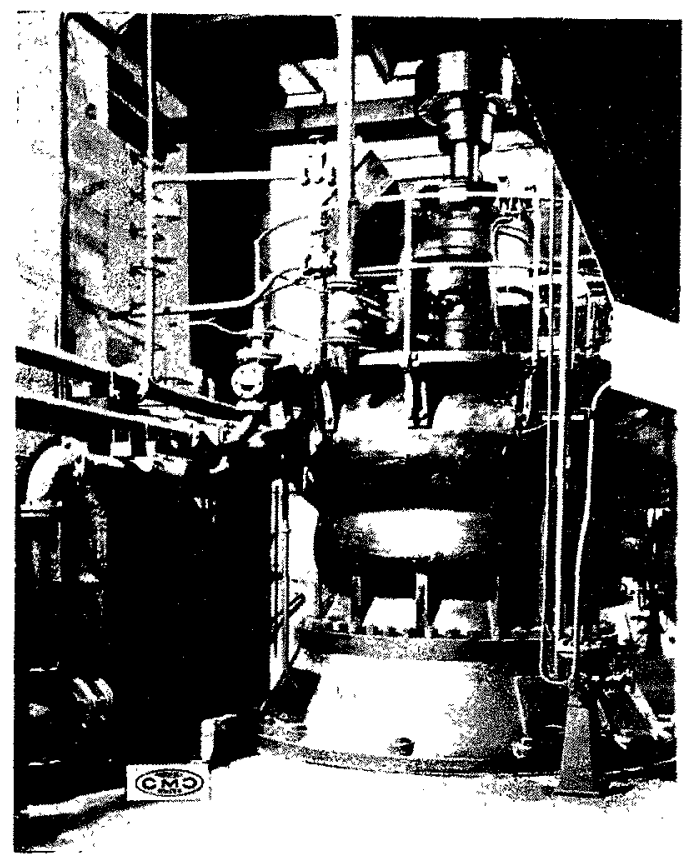

Fig. 27.- Usine de Waggital (Suisse). Groupe moto-pompe de $5.500 \mathrm{CV}$ à axe vertical.

La mise en route des 2 premières unités qui a eu lieu au cours de ces dernières semaines s'est effectuée sans le moindre incident avec une régularité parfaite.

Des résultats aussi encourageants nous permettent donc d'envisager pour lavenir des installations de plus grande envergure encore.

Le coût relativement faible d'une installation de pompe, comparé aux énormes immobilisations de capitaux qu'entrainent des aménagements de barrage et de conduites forcées comme celles de Belleville ou de Wàggital ne permet pas de s'arrêter sérieusement à la question dépense de premier établissement On admet couramment par exemple qu'une installation lyydroélectrique de montagne con̂te aujourd'hui de 3.500 à $4.000 \mathrm{fr}$. environ le kilowatt installé, soit environ 20 millions pour une usine de 5.000 kilowatts. L'addition d'une pompe centrifuge à cette installation ne majorera certainement pas la dépense de plus de 500.000 francs. Si l'on songe qu'elle permet, comme à Belleville, de récupérer anmuellement 17 millions de Kwh, on voit immédiatement que cette addition à l'installatio presque immédiatement amortie.

Dans un autre ordre d'idées, on peut se demander dans $q$ mesure il est intéressant, dans un réseau hydroélectrique portant, entr'autres usines génératrices, une usine de pl à régime sensiblement constant, de réaliser l'appoint à l' d'une centrale de montagne avec accumulation, ou à l'aide $\mathrm{d}$ Centrale de secours avec moteur Diesel.

C'est évidemment une question d'espèces qui dépend es tiellement des capitaux à immobiliser sur l'installation hyd lique, du loyer de l'argent, et du prix de l'huile lourde néces au fonctionnement du Diesel. A l'inverse de l'installation hyd

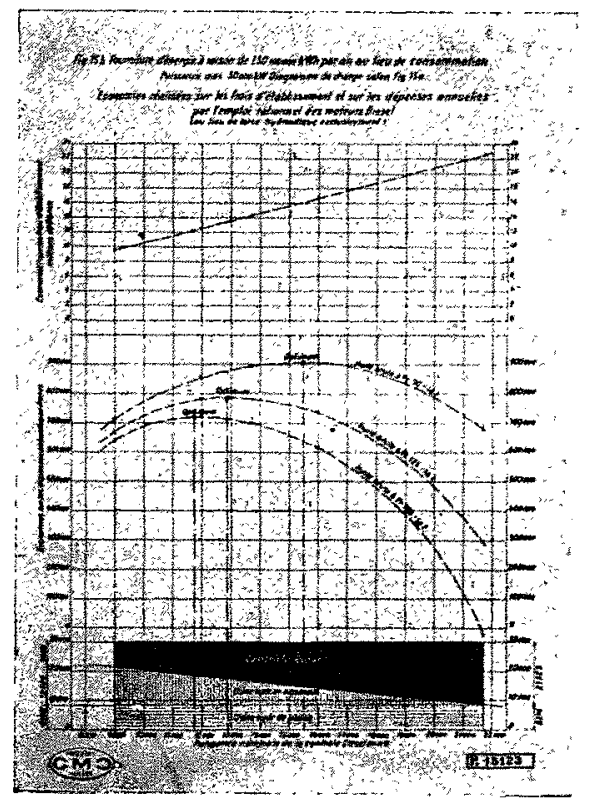

Fig. 25.- Diagrammes d'emploi continu d'une Usine hydroélectr avec accumulateur et d'une centrale Diesel.

lique, les frais d'installation de la Centrale Diesel intervien dans une faible mesure dans le prix de revient du Kilow heure aiors que les frais d'exploitation interviennent d'une $f$ importante en raison du prix de l'huile.

On pourra, dans chaque cas particulier, établir un diagra dans le genre de celui (photo 25) qui a pour but de faire ress l'économie que l'installation d'un Diesel permet de réaliser l'installation purement hydraulique.

Le diagramme est établi pour une puissance totale de 30.00 installés comportant $8.275 \mathrm{Kw}$ en une usine de plaine à rẻ constant et $21.725 \mathrm{Kw}$ à répartir entre une usine hydrau avec accumulation et une Centrale Diesel. En abcisses on $p$ la puissance attribuée à la Centrale Diesel et en ordonnée l' nomie réalisée par cette installation sur l'installation pure hydraulique. On pourra tracer différentes courbes sui le prix de la tome de combustible; naturellement, le $\mathrm{p}$ d'économie optima se déplace en faveur de la solution hyd lique au fur et à mesure qu'augmente la tomne de combustibl 